\title{
Global stability of a fractional-order logistic growth model with infectious disease
}

\section{Hasan S. Panigoro and Emli Rahmi}

To cite this article:

H. S. Panigoro and E. Rahmi,"Global stability of a fractional-order logistic growth model with infectious disease", Jambura J. Biomath, vol. 1, no. 2, pp. 49-56, 2020

DOI: https://doi.org/10.34312/jjbm.v1i2.8135

(C) 2020 Author(s).

\section{Articles You may be interested in}

A stage-structure Rosenzweig-MacArthur model with effect of prey refuge

L. K. Beay and M. Saija

http://dx.doi.org/10.34312/jjbm.v1i1.6891

Analisis kestabilan model predator-prey dengan infeksi penyakit pada prey dan pemanenan proporsional pada predator

S. Maisaroh, Resmawan, and E. Rahmi

http://dx.doi.org/10.34312/jjbm.v1i1.5948

Bifurkasi Hopf pada model Lotka-Volterra orde-fraksional dengan Efek Allee aditif pada predator

H. S. Panigoro and D. Savitri

http://dx.doi.org/10.34312/jjbm.v1i1.6908

Parameters estimation of generalized Richards model for COVID-19 cases in Indonesia using genetic algorithm

M. Rayungsari, M. Aufin, and N. Imamah

http://dx.doi.org/10.34312/jjbm.v1i1.6910

Revitalisasi danau Limboto dengan pengerukan endapan di danau: pemodelan, analisis, dan simulasinya

S. L. Mahmud, N. Achmad, and H. S. Panigoro

http://dx.doi.org/10.34312/jjbm.v1i1.6945 


\title{
Global stability of a fractional-order logistic growth model with infectious disease
}

\author{
Hasan S. Panigoro ${ }^{1, *}$, Emli Rahmi ${ }^{1}$ \\ ${ }^{1}$ Department of Mathematics, State University of Gorontalo, Bone Bolango 96119, Indonesia \\ *Corresponding author. Email: hspanigoro@ung.ac.id
}

\begin{abstract}
Infectious disease has an influence on the density of a population. In this paper, a fractional-order logistic growth model with infectious disease is formulated. The population grows logistically and divided into two compartments i.e. susceptible and infected populations. We start by investigating the existence, uniqueness, non-negativity, and boundedness of solutions. Furthermore, we show that the model has three equilibrium points namely the population extinction point, the disease-free point, and the endemic point. The population extinction point is always a saddle point while others are conditionally asymptotically stable. For the non-trivial equilibrium points, we successfully show that the local and global asymptotic stability have the similar properties. Especially, when the endemic point exists, it is always globally asymptotically stable. We also show the existence of forward bifurcation in our model. We portray some numerical simulations consist of the phase portraits, time series, and a bifurcation diagram to validate the analytical findings.
\end{abstract}

Keywords: Dynamics, Fractional-Order, Logistic Growth, Infectious Disease

\section{Introduction}

The logistic growth model is first proposed by a Belgian mathematician called Pierre François Verhulst [1], which given by a first-order differential equation

$$
\frac{d N}{d t}=r N\left(1-\frac{N}{K}\right),
$$

where $r$ is the intrinsic growth rate and $K$ is the environmental carrying capacity of the population. The logistic growth model is considered more realistic rather than the exponential growth model proposed by Malthus [2]. To achieve a more specific condition of natural phenomena, the logistic growth model is modified such as Richards model [3], Blumberg model [4], and Tsoularis model [5]. Some modifications are also done to facilitate the biological behavior of populations for instance: the Allee effect [6-8] and feedback control [9, 10]. It is also applied in interaction between populations in predator-prey schemes as in [11-16]. Nowadays, the logistic model and its modifications are used to modeling and predicting the infected population of new coronavirus 2019 (COVID-19), see [17-21].

In this paper, we modify the logistic growth model (1) by assuming the population is infected by a disease. We divide the population $N(t)$ into two compartments namely susceptible population $S(t)$ and infected population $I(t)$. We also assume that the infection rate is bilinear and the infected population may recover by its immune system. This model is given by a first-order differential equations as follows.

$$
\begin{aligned}
& \frac{d S}{d t}=r S\left(1-\frac{S+I}{K}\right)-\beta S I+\omega I, \\
& \frac{d I}{d t}=\beta S I-(\omega+\delta) I,
\end{aligned}
$$

where $\beta$ is the infection rate, $\omega$ is the recovery rate, and $\delta$ is the death rate causes by disease. It is clear that the total population $N(t)$ satisfies $N(t)=S(t)+I(t)$. Some similar works are done by scholars [10, 22-29]. The novelty of our model compared with their models lies on the utilization of logistic growth rate rather than the constant growth rate.

To attain more realistic model, we apply the fractional-order derivative as the operator. The model with fractional-order derivative describe a preferable biological condition rather than the first-order derivative since 
this operator has capability in involving all previous condition to express the state condition which called the memory effect, see [30-35]. By replacing the first-order derivative at the left hand side of model (2) with the fractional-order derivative and redefine some parameters in similar way with [32] to obtain the same dimension of (time) $)^{\alpha}$, we get

$$
\begin{aligned}
& { }^{C} \mathcal{D}_{t}^{\alpha} S=r S\left(1-\frac{S+I}{K}\right)-\beta S I+\omega I=G_{1}(N), \\
& { }^{C} \mathcal{D}_{t}^{\alpha} I=\beta S I-(\omega+\delta) I=G_{2}(N),
\end{aligned}
$$

where ${ }^{C} \mathcal{D}_{t}^{\alpha}$ is Caputo fractional-order derivative define by

$$
{ }^{C} \mathcal{D}_{t}^{\alpha} f(t)=\frac{1}{\Gamma(1-\alpha)} \int_{0}^{t}(t-s)^{-\alpha} f^{\prime}(\tau) d \tau
$$

with $t \geq 0, f \in C^{n}([0,+\infty), \mathbb{R})$, and $\Gamma(\cdot)$ is a Gamma function [36].

This paper is organized as follows. To ensure the model (3) meets the expected biological conditions, the existence, uniqueness, non-negativity, and boundedness are given in Sections 2 and 3. In Section 4, we study the dynamics of model (3) including the existence of equilibrium points, their local and global stability, and the existence of forward bifurcation. Finally, we close our works by giving some concluding remarks in Section 5 .

\section{Existence and Uniqueness}

Suppose that $\Theta:=\left\{(S, I) \in \mathbb{R}^{2}: \max (|S|,|I|) \leq \sigma\right\}$. We will show that for each initial values in $\Theta$, the model (3) has a unique solution in the region $\Theta \times(0, T], T<\infty$. The similar approach used in [31, 32] is employed. We consider a mapping $G(N)=\left(G_{1}(N), G_{2}(N)\right)$. For any $N=(S, I), \bar{N}=(\bar{S}, \bar{I}), N, \hat{N} \in \Theta$, we acquire

$$
\begin{aligned}
\|G(N)-G(\bar{N})\|= & \left|G_{1}(N)-G_{1}(\bar{N})\right|+\left|G_{2}(N)-G_{2}(\bar{N})\right| \\
= & \left|\left(r S\left(1-\frac{S+I}{K}\right)-\beta S I+\omega I\right)-\left(r \bar{S}\left(1-\frac{\bar{S}+\bar{I}}{K}\right)-\beta \bar{S} \bar{I}+\omega \bar{I}\right)\right|+ \\
& |(\beta S I-(\omega+\delta) I)-(\beta \bar{S} \bar{I}-(\omega+\delta) \bar{I})| \\
= & \left|r(S-\bar{S})-\frac{r}{K}\left(S^{2}-\bar{S}^{2}\right)-\left(\frac{r}{K}+\beta\right)(S I-\bar{S} \bar{I})+\omega(I-\bar{I})\right|+ \\
& |\beta(S I-\bar{S} \bar{I})-(\omega+\delta)(I-\bar{I})| \\
\leq & r|S-\bar{S}|+\frac{r}{K}\left|S^{2}-\bar{S}^{2}\right|+\left(\frac{r}{K}+\beta\right)|S I-\bar{S} \bar{I}|+\omega|I-\bar{I}|+ \\
& \beta|S I-\bar{S} \bar{I}|+(\omega+\delta)|I-\bar{I}| \\
= & r|S-\bar{S}|+\frac{r}{K}|S+\bar{S}||S-\bar{S}|+\left(\frac{r}{K}+2 \beta\right)|I(S-\bar{S})+\bar{S}(I-\bar{I})|+(2 \omega+\delta)|I-\bar{I}| \\
\leq & r|S-\bar{S}|+\frac{2 \sigma r}{K}|S-\bar{S}|+\left(\frac{r}{K}+2 \beta\right) \sigma|S-\bar{S}|+\left(\frac{r}{K}+2 \beta\right) \sigma|I-\bar{I}|+(2 \omega+\delta)|I-\bar{I}| \\
= & \left(r+\frac{3 \sigma r}{K}+2 \sigma \beta\right)|S-\bar{S}|+\left(\frac{\sigma r}{K}+2 \sigma \beta+2 \omega+\delta\right)|I-\bar{I}| \\
\leq & L\|N-\bar{N}\|,
\end{aligned}
$$

where $L=\max \left\{r+\frac{3 \sigma r}{K}+2 \sigma \beta, \frac{\sigma r}{K}+2 \sigma \beta+2 \omega+\delta\right\}$, and hence $G(N)$ satisfies the Lipschitz condition. Obeying Theorem 3.7 in [37], the existence and uniqueness of solution of model (3) is satisfied. Therefore, the following theorem is preserved.

Theorem 1. For each non-negative initial condition in $\Theta$, there exists a unique solution of model (3) in the region $\Theta \times$ $(0, T]$.

\section{Non-negativity and Boundedness}

The non-negativity and boundedness of solutions of model (3) are given by the following theorem.

Theorem 2. For each non-negative initial conditions, the solutions of model (3) are always non-negative and uniformly bounded. 
proof. By using conformable manner as in [31] and applying Lemma 2 in [32], it can be clarified that the solutions are non-negative if the initial conditions are also non-negative. Furthermore, we give the proof of the boundedness of solutions. We confirm that the total population $N(t)$ satisfies $N(t)=S(t)+I(t)$, and hence we get

$$
\begin{aligned}
{ }^{C} \mathcal{D}_{t}^{\alpha} N(t)+\delta N(t) & =r S\left(1-\frac{S+I}{K}\right)-\beta S I+\omega I+\beta S I-(\omega+\delta) I+\delta S+\delta I \\
& =(r+\delta) S-\frac{r S^{2}}{K}-\frac{r S I}{K} \\
& =-\frac{r}{K}\left(S-\frac{(r+\delta) K}{2 r}\right)^{2}+\frac{(r+\delta)^{2} K}{4 r}-\frac{r S I}{K} \\
& \leq \frac{(r+\delta)^{2} K}{4 r} .
\end{aligned}
$$

Based on Lemma 3 in [38], we have

$$
N(t) \leq\left(N(0)-\frac{(r+\delta)^{2} K}{4 r}\right) E_{\alpha}\left[-\delta t^{\alpha}\right]+\frac{(r+\delta)^{2} K}{4 r},
$$

where $E_{\alpha}$ is mittag-leffler function. Therefore, we have $N(t) \leq \frac{(r+\delta)^{2} K}{4 r}$ for $t \rightarrow \infty$, which convince all solutions are confined to the region $\Psi$ where

$$
\Psi:=\left\{(x, y) \in \mathbb{R}_{+}^{2}: S+I \leq \frac{(r+\delta)^{2} K}{4 r}+\varepsilon, \varepsilon>0\right\} .
$$

Consequently, all solutions of model (3) are uniformly bounded.

\section{Equilibrium Points and Their Stability}

We acquire the equilibrium point by solving $G_{1}(N)=G_{2}(N)=0$. In consequence, we obtain three equilibrium points i.e the population extinction point $\Omega_{0}=(0,0)$, the disease-free point $\Omega_{1}=(K, 0)$, and the endemic equilibrium point $\Omega_{2}=\left(\frac{K}{R_{0}},\left(1-\frac{1}{R_{0}}\right) \frac{r K}{r+\delta R_{0}}\right)$ where $R_{0}=\frac{\beta K}{\omega+\delta}$. Furthermore, the dynamics of model (3) consist of the local and global stability are shown by the following theorems.

Theorem 3. The population extinction point $\Omega_{0}=(0,0)$ is always a saddle point.

proof. We compute the Jacobian matrix of model (3) at $\Omega_{0}$. Thus, we have

$$
J\left(\Omega_{0}\right)=\left[\begin{array}{cc}
r & \omega \\
0 & -(\omega+\delta)
\end{array}\right],
$$

which gives the eigenvalues: $\lambda_{1}=r$ and $\lambda_{2}=-(\omega+\delta)$. We confirm that $\left|\arg \left(\lambda_{1}\right)\right|<\alpha \pi / 2$ and $\left|\arg \left(\lambda_{2}\right)\right|>$ $\alpha \pi / 2$, which ensures that $\Omega_{0}$ is always a saddle point, see Theorem 3 in [32].

Theorem 4. If $R_{0}<1$ then the disease-free point $\Omega_{1}=(K, 0)$ is asymptotically stable both locally and globally. Otherwise, it is a saddle point.

proof. We start by identify the local stability of $\Omega_{1}$. For $\Omega_{1}=(K, 0)$, the Jacobian matrix of model (3) is

$$
J\left(\Omega_{1}\right)=\left[\begin{array}{cc}
-r & -\left(\frac{r}{K}+\beta\right) K+\omega \\
0 & (\omega+\delta)\left(R_{0}-1\right)
\end{array}\right] .
$$

From (6), we have the eigenvalues: $\lambda_{1}=-r$ which gives $\left|\arg \left(\lambda_{1}\right)\right|>\alpha \pi / 2$ and $\lambda_{2}=(\omega+\delta)\left(R_{0}-1\right)$ where $\left|\arg \left(\lambda_{2}\right)\right|$ depends on the value of $R_{0}$. If $R_{0}<1$ then $\left|\arg \left(\lambda_{2}\right)\right|>\alpha \pi / 2$, and If $R_{0}>1$ then $\left|\arg \left(\lambda_{2}\right)\right|<\alpha \pi / 2$. By applying Theorem 3 in [32], the local dynamics of model (3) is emphasized. 
Now, we give the proof that the global stability of $\Omega_{1}$ has the similar properties as its local one. We define a positive Lyapunov function as follows.

$$
\Phi_{1}(S, I)=\left[S-K-K \ln \frac{S}{K}\right]+\frac{(r+\beta K) R_{0}}{\beta K} I .
$$

According to Lemma 3.1 in [39], the fractional-order derivative of (7) is given by

$$
\begin{aligned}
{ }^{C} \mathcal{D}_{t}^{\alpha} \Phi_{1}(S, I) & \leq\left(\frac{S-K}{S}\right){ }^{C} \mathcal{D}_{t}^{\alpha} S+\frac{(r+\beta K) R_{0}}{\beta K}{ }^{C} \mathcal{D}_{t}^{\alpha} I \\
& =\left(\frac{S-K}{S}\right)\left(r S\left(1-\frac{S+I}{K}\right)-\beta S I+\omega I\right)+\frac{(r+\beta K) R_{0}}{\beta K}(\beta S I-(\omega+\delta) I) \\
& =(S-K)\left(r-\frac{r S+r I}{K}-\beta I+\omega \frac{I}{S}\right)+\frac{(r+\beta K) R_{0} S I}{K}-\frac{(\omega+\delta)(r+\beta K) R_{0}}{\beta K} I \\
& =\left(2 r S-r K-\frac{r S^{2}}{K}-\frac{r S I}{K}+r I-\beta S I+\beta K I+\omega I-\frac{\omega K I}{S}\right)+\frac{(r+\beta K) R_{0} S I}{K}-(r+\beta K) I \\
& =-\frac{r S^{2}}{K}+2 r S-r K-\left(\frac{r}{K}+\beta-\frac{(r+\beta K) R_{0}}{K}\right) S I-\frac{\omega K I}{S} \\
& =-\frac{r}{K}(S-K)^{2}-\frac{\omega K I}{S}-\frac{\left(1-R_{0}\right)(r+\beta K) S I}{K} .
\end{aligned}
$$

Since $R_{0}<1$, we conclude that ${ }^{C} \mathcal{D}_{t}^{\alpha} \Phi_{1}(S, I) \leq 0$ for all $(S, I) \in \mathbb{R}_{+}^{2}$. We also ensure that ${ }^{C} \mathcal{D}_{t}^{\alpha} \Phi_{1}(S, I)=0$ implies that $(S, I)=(K, 0)$. Therefore, the only invariant set on which ${ }^{C} \mathcal{D}_{t}^{\alpha} \Phi_{1}(S, I)=0$ is the singleton $\left\{\Omega_{1}\right\}$. Obeying Lemma 4.6 in [40], the disease-free point $\Omega_{1}=(K, 0)$ is globally asymptotically stable.

Remark 1. From Theorem 4, we conclude that if $R_{0}<1$ then $\Omega_{1}$ is locally and globally asymptotically stable. For all initial conditions, the disease will become extinct, the density of population will eventually increases, and tends to the environmental carrying capacity.

Theorem 5. The endemic equilibrium point $\Omega_{2}=\left(\frac{K}{R_{0}},\left(1-\frac{1}{R_{0}}\right) \frac{r K}{r+\delta R_{0}}\right)$ is always asymptotically stable both locally and globally.

proof. Suppose that

$$
\begin{aligned}
& \xi_{1}=\frac{R_{0} \xi_{2}}{\beta K}\left[1-\frac{r+(\omega+\delta) R_{0}}{r+\delta R_{0}}\right]-\frac{r}{R_{0}}, \\
& \xi_{2}=\left(1-\frac{1}{R_{0}}\right) \frac{\beta r K}{R_{0}} .
\end{aligned}
$$

It is easy to verify that $\xi_{1}<0$ and $\xi_{2}>0$. By evaluating Jacobian matrix of model (3) at $\Omega_{2}$, we acquire

$$
J\left(\Omega_{2}\right)=\left[\begin{array}{cc}
\xi_{1} & -\frac{r+\delta R_{0}}{R_{0}} \\
\frac{R_{0} \xi_{2}}{r+\delta R_{0}} & 0
\end{array}\right],
$$

and obtain a pair of eigenvalues: $\lambda_{1}=\frac{\xi_{1}}{2} \pm \frac{1}{2} \sqrt{\xi_{1}^{2}-4 \xi_{2}}$. For $\xi_{1}^{2}<4 \xi_{2}$, those eigenvalues are a pair of complex conjugate. Because the real part $\frac{\xi_{1}}{2}<0$, then we have $\left|\arg \left(\lambda_{1,2}\right)\right|>\frac{\alpha \pi}{2}$. Therefore, $\Omega_{2}$ is locally asymptotically stable. For $\xi_{1}^{2} \geq 4 \xi_{2}$, according to the Routh-Hurwitz theorem for Caputo fractional order [41], $\Omega_{2}$ is locally asymptotically stable if $\xi_{1}<0$. Since $\xi_{1}<0$, the endemic point $\Omega_{2}$ is always locally asymptotically stable. Now we will show the global stability of $\Omega_{2}$ also has the similar properties with the local one. Let $S^{*}=\frac{K}{R_{0}}$ and $I^{*}=\left(1-\frac{1}{R_{0}}\right) \frac{r K}{r+\delta R_{0}}$. Therefore, we define a positive Lyapunov function as follows.

$$
\Phi_{2}(S, I)=\left[S-S^{*}-S^{*} \ln \frac{S}{S^{*}}\right]+\kappa\left[I-I^{*}-I^{*} \ln \frac{I}{I^{*}}\right],
$$


Conforming Lemma 3.1 in [39], we obtain

$$
\begin{aligned}
{ }^{C} \mathcal{D}_{t}^{\alpha} \Phi_{2}(S, I) & \leq\left(\frac{S-S^{*}}{S}\right){ }^{C} \mathcal{D}_{t}^{\alpha} S+\kappa\left(\frac{I-I^{*}}{I}\right){ }^{C} \mathcal{D}_{t}^{\alpha} I \\
& =\left(\frac{S-S^{*}}{S}\right)\left(r S\left(1-\frac{S+I}{K}\right)-\beta S I+\omega I\right)+\kappa\left(\frac{I-I^{*}}{I}\right)(\beta S I-(\omega+\delta) I) \\
& =\left(S-S^{*}\right)\left(r\left(1-\frac{S+I}{K}\right)-\beta I+\frac{\omega I}{S}\right)+\kappa\left(I-I^{*}\right)(\beta S-(\omega+\delta)) \\
& =\left(S-S^{*}\right)\left(r-\frac{r S}{K}-\frac{r I}{K}-\beta I+\frac{\omega I}{S}\right)+\kappa\left(I-I^{*}\right)(\beta S-(\omega+\delta)) \\
& =\left(S-S^{*}\right)\left(\frac{r S^{*}}{K}+\frac{r I^{*}}{K}+\beta I^{*}-\frac{\omega I^{*}}{S^{*}}-\frac{r S}{K}-\frac{r I}{K}-\beta I+\frac{\omega I}{S}\right)+\kappa \beta\left(I-I^{*}\right)\left(S-S^{*}\right) \\
& =\left(S-S^{*}\right)\left(-\frac{r}{K}\left(S-S^{*}\right)-\frac{r}{K}\left(I-I^{*}\right)-\beta\left(I-I^{*}\right)-\frac{\omega I^{*}}{S^{*}}+\frac{\omega I}{S}\right)+\kappa \beta\left(I-I^{*}\right)\left(S-S^{*}\right) \\
& =-\frac{r}{K}\left(S-S^{*}\right)^{2}-\left(\frac{r}{K}+\beta-\kappa \beta\right)\left(S-S^{*}\right)\left(I-I^{*}\right)-\omega\left(S-S^{*}\right)\left(\frac{-S\left(I-I^{*}\right)+I\left(S-S^{*}\right)}{S^{*} S}\right) \\
& =-\left(\frac{r}{K}+\frac{\omega I}{S^{*} S}\right)\left(S-S^{*}\right)^{2}-\left(\frac{r+\beta K}{K}-\kappa \beta-\frac{\omega}{S^{*}}\right)\left(S-S^{*}\right)\left(I-I^{*}\right)
\end{aligned}
$$

By choosing $\kappa=\frac{r+\delta R_{0}}{\beta K}$, we get

$$
{ }^{C} \mathcal{D}_{t}^{\alpha} \Phi_{2}(S, I) \leq-\left(\frac{r}{K}+\frac{\omega I}{S^{*} S}\right)\left(S-S^{*}\right)^{2} .
$$

Therefore, ${ }^{C} \mathcal{D}_{t}^{\alpha} \Phi_{2}(S, I) \leq 0$ for all $(S, I) \in \mathbb{R}_{+}^{2}$, and ${ }^{C} \mathcal{D}_{t}^{\alpha} \Phi_{2}(S, I)=0$ implies that $(S, I)=\left(S^{*}, I^{*}\right)$ which means the singleton $\left\{\Omega_{2}\right\}$ is the only invarian set on which ${ }^{C} \mathcal{D}_{t}^{\alpha} \Phi_{2}(S, I)=0$. By utilizing Lemma 4.6 in [40], the endemic point $\Omega_{2}$ is always globally asymptotically stable.

Remark 2. According to Theorem 5, if $R_{0}>1$, then $\Omega_{2}$ is locally and globally asymptotically stable. For all positive initial conditions, the disease always exists as $t \rightarrow \infty$ and convergent to an endemic point $\Omega_{2}$. Although the disease will not extinct, when $R_{0}>1$ the infected population has a bounded density.

Theorem 6. The equilibrium point $\Omega_{1}$ undergoes a forward bifurcation when $R_{0}$ passes through the critical point $R_{0}^{*}=1$.

proof. When $R_{0}<R_{0}^{*}$, the disease-free point $\Omega_{1}$ is the only non-trivial equilibrium point of model (3) which is always asymptotically stable. When $R_{0}>R_{0}^{*}, \Omega_{1}$ losses its stability and an always asymptotically stable $\Omega_{2}$ occurs simultaneously. By considering $I^{*}=\left(1-\frac{1}{R_{0}}\right) \frac{r K}{r+\delta R_{0}}$, we confirm that the increase of $R_{0}$ means the increase of the infected populations.

Remark 3. Theorem 6 and the value of $I^{*}$ in Theorem 5 show us that $R_{0}$ has an important rule in determining the existence of the infected population. If $R_{0}$ is enlarged, the intrinsic growth rate of infected populations is increased. In some literature, $R_{0}$ is called the ratio reproduction number, which is not discussed in detail in this article.

\section{Numerical Simulations}

Some numerical simulations are demonstrated to confirm all analytical results. The predictor-corrector scheme for Caputo fractional-order derivative developed by Diethelm [42] is applied. Since the model is studied qualitatively and there are limitations in getting the field data, we take the suppositional values as the parameters. By fixing parameter values: $r=0.5, K=5, \Omega=0.2$, and $\delta=0.1$, and varying the parameter of infection rate $\beta$ in interval $0<\beta<0.24$ (so that $0<R_{0}<4$ ) we plot the bifurcation diagram in Figure 1 . The disease-free point $\Omega_{1}$ which is asymptotically stable when $R_{0}<1$ is separated into two equilibrium points namely the unstable $\Omega_{1}$ and the asymptotically stable endemic point $\Omega_{2}$ when $R_{0}>1$. $\Omega_{1}$ losses its stability when $\beta$ passes through $\beta^{*}=0.06$ or $R_{0}$ passes through $R_{0}^{*}=1$, and an asymptotically stable equilibrium point appears in the interior simultaneously. Based on Theorem 6, this phenomenon is called forward bifurcation. 

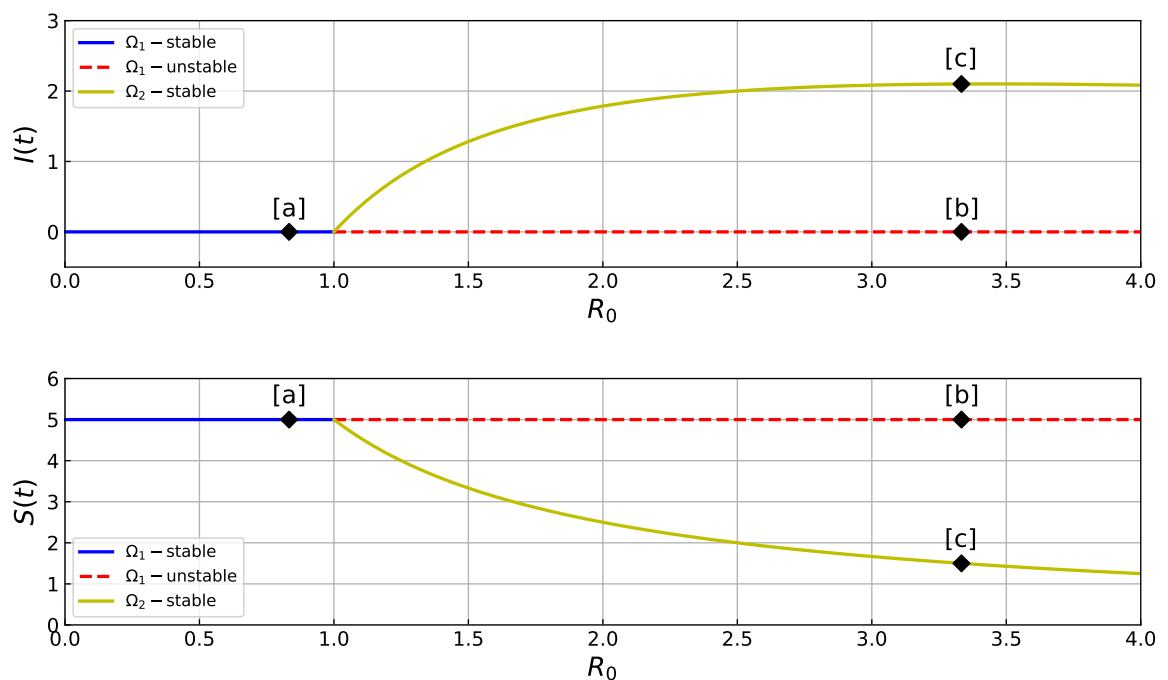

Figure 1. Bifurcation diagram of model (3) driven by $\beta$ with paramater values: $r=0.5, K=5, \Omega=0.2$, and $\delta=0.1$.

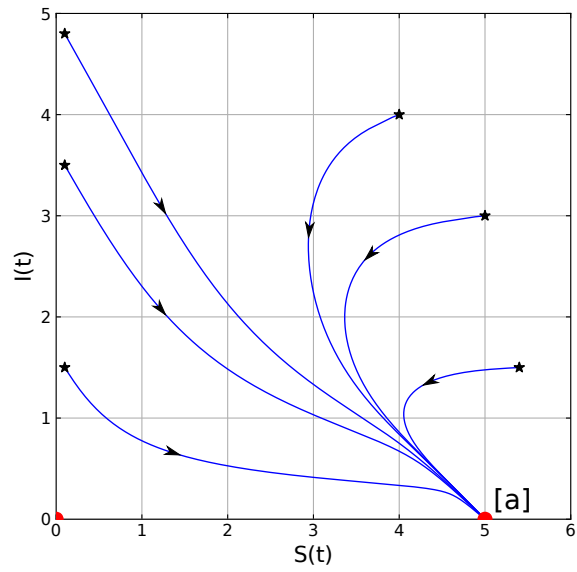

(a) Phase portrait

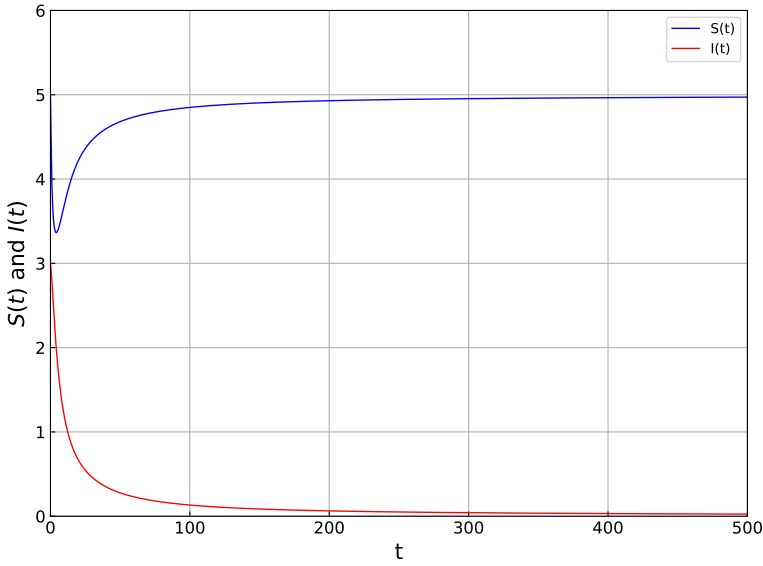

(b) Time series

Figure 2. Numerical simulation of model (3) with parameter values: $r=0.5, K=5, \beta=0.05 \Omega=0.2$, and $\delta=0.1$

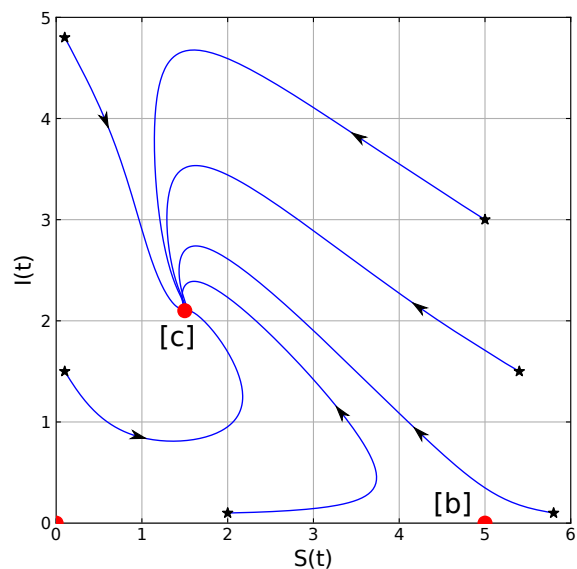

(a) Phase portrait

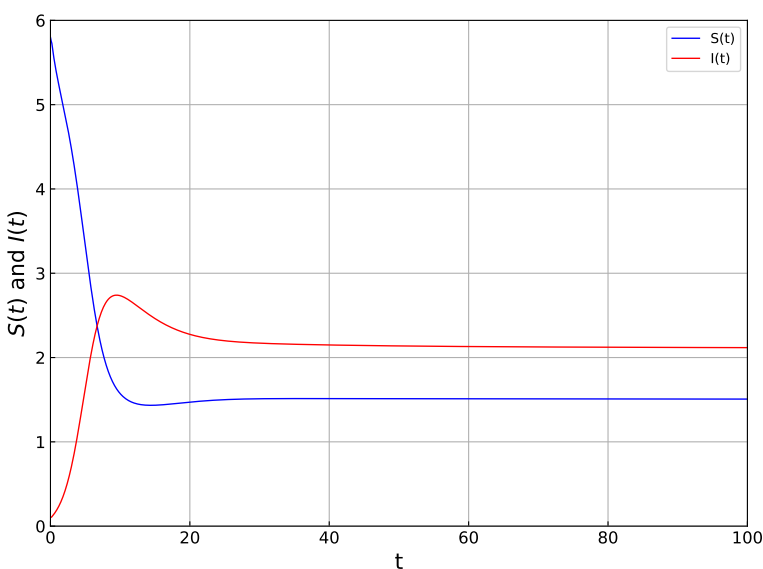

(b) Time series

Figure 3. Numerical simulation of model (3) with parameter values: $r=0.5, K=5, \beta=0.2 \Omega=0.2$, and $\delta=0.1$ 
To show the dynamical behavior of model (3) when $R_{0}<1$ and $R_{0}>1$, we set $\beta=0.05$ (or $R_{0}=0.83333$ ) and $\beta=0.2$ (or $R_{0}=3.33333$ ), respectively. When $R_{0}=0.83333$, we have an equilibrium point which given by label $[a]=(5,0)$, see Figure 1 . The equilibrium point [a] is asymptotically stable both locally and globally as proven in Theorem 4, and shown in Figure 2. For $R_{0}=3.33333$, we have two equilibrium points labeled by [b] and [c], see Figure 1. The equilibrium point [b] is unstable point while the equilibrium point [c] is globally asymptotically stable. The phaseportrait and the time series are shown in Figure 3. From those numerical simulations, we conclude that when $R_{0}<1$, the disease won't spread, and when $R_{0}>1$, the disease will spread and convergent to a constant value. The disease is still exists for $t \rightarrow \infty$ but the density of infected population is bounded.

\section{Conclusion}

The dynamics of a fractional-order logistic growth model with infectious disease has been investigated. We show analytically that the model has atmost three equilibrium points i.e: the population extinction point which is always exists and a saddle point, the disease-free point which is always exists, asymptotically stable when $R_{0}<1$ and a saddle point when $R_{0}>1$, and the endemic point which is exists and asymptotically stable when $R_{0}>1$. We succesfully proof that the global stability has the similar properties as the local one for each nontrivial equilibrium point. We also show there exists a forward bifurcation driven by $\beta$ or $R_{0}$. From biological point of view, if $R_{0}<1$ the disease will extinct, and if $R_{0}>1$, the disease will spreads. Although the disease is spreading, the disease will bounded an confine to a region.

\section{References}

[1] P. F. Verhulst, “Notice sur la loi que la population poursuit dans son accroissement," Correspondance mathématique et physique, vol. 10, pp. 113-121, 1838.

[2] T. R. Malthus, An essay on the principle of population. (Reprinted from 1798 edition, Johnson, London), reprinted ed. Michigan, USA: University of Michigan, 1798.

[3] F. J. Richards, "A Flexible growth function for empirical use," Journal of Experimental Botany, vol. 10, no. 29, pp. 290-300, 1959.

[4] A. A. Blumberg, "Logistic growth rate functions," Journal of Theoretical Biology, vol. 21, no. 1, pp. 42-44, 1968.

[5] A. Tsoularis and J. Wallace, "Analysis of logistic growth models," Mathematical Biosciences, vol. 179, no. 1, pp. $21-55,2002$.

[6] Y. Kang and O. Udiani, "Dynamics of a single species evolutionary model with Allee effects," Journal of Mathematical Analysis and Applications, vol. 418, no. 1, pp. 492-515, 2014.

[7] S. Abbas, M. Banerjee, and S. Momani, "Dynamical analysis of fractional-order modified logistic model," Computers and Mathematics with Applications, vol. 62, no. 3, pp. 1098-1104, 2011.

[8] J. Leonel Rocha and A. K. Taha, "Allee's effect bifurcation in generalized logistic maps," International Journal of Bifurcation and Chaos, vol. 29, no. 3, pp. 1-19, 2019.

[9] Q. Lin, "Stability analysis of a single species logistic model with Allee effect and feedback control," Advances in Difference Equations, vol. 2018, no. 1, pp. 1-13, 2018.

[10] M. T. Hoang and O. F. Egbelowo, "Numerical dynamics of nonstandard finite difference schemes for a Logistics model with feedback control," Annali dell'Universita di Ferrara, vol. 66, no. 1, pp. 51-65, 2020.

[11] H. S. Panigoro, A. Suryanto, W. M. Kusumahwinahyu, and I. Darti, "Dynamics of a fractional-order predator-prey model with infectious diseases in prey," Communication in Biomathematical Sciences, vol. 2, no. 2, p. 105, 2019.

[12] A. Suryanto, I. Darti, H. S. Panigoro, and A. Kilicman, “A fractional-order predator-prey model with ratio-dependent functional response and linear harvesting," Mathematics, vol. 7, no. 11, pp. 1-13, 2019.

[13] H. S. Panigoro, A. Suryanto, W. M. Kusumawinahyu, and I. Darti, "Continuous threshold harvesting in a gause-type predator-prey model with fractional-order," AIP Conference Proceedings, vol. 2264, no. 1, p. 040001, 2020.

[14] H. S. Panigoro, E. Rahmi, N. Achmad, and S. L. Mahmud, "The influence of additive Allee effect and periodic harvesting to the dynamics of Leslie-Gower predator-prey model," Jambura Journal of Mathematics, vol. 2, no. 2, pp. 87-96, 2020.

[15] D. Mukherjee and C. Maji, “Bifurcation analysis of a Holling type II predator-prey model with refuge," Chinese Journal of Physics, vol. 65, no. February, pp. 153-162, 2020.

[16] L. K. Beay and M. Saija, "A stage-structure Rosenzweig-MacArthur model with effect of prey refuge," Jambura Journal of Biomathematics (JJBM), vol. 1, no. 1, pp. 1-7, 2020.

[17] B. Malavika, S. Marimuthu, M. Joy, A. Nadaraj, E. S. Asirvatham, and L. Jeyaseelan, "Forecasting COVID-19 epidemic in India and high incidence states using SIR and logistic growth models," Clinical Epidemiology and Global Health, no. May, pp. 1-8, 2020.

[18] P. Wang, X. Zheng, J. Li, and B. Zhu, "Prediction of epidemic trends in COVID-19 with logistic model and machine learning technics," Chaos, Solitons and Fractals, vol. 139, p. 110058, 2020.

[19] N. Nuraini, K. Khairudin, and M. Apri, "Modeling simulation of COVID-19 in Indonesia based on early endemic data," Communication in Biomathematical Sciences, vol. 3, no. 1, pp. 1-8, 2020.

[20] C. Y. Shen, "Logistic growth modelling of COVID-19 proliferation in China and its international implications," International Journal of Infectious Diseases, vol. 96, pp. 582-589, 2020. 
[21] M. Rayungsari, M. Aufin, and N. Imamah, "Parameters estimation of generalized Richards model for COVID-19 cases in Indonesia using genetic algorithm," Jambura Journal of Biomathematics (JJBM), vol. 1, no. 1, pp. 25-30, 2020.

[22] M. A. Abdelaziz, A. I. Ismail, F. A. Abdullah, and M. H. Mohd, "Bifurcations and chaos in a discrete SI epidemic model with fractional order," Advances in Difference Equations, vol. 2018, no. 1, 2018.

[23] S. Iwami, Y. Takeuchi, and X. Liu, "Avian-human influenza epidemic model," Mathematical Biosciences, vol. 207, no. 1, pp. 1-25, 2007.

[24] F. M. Hilker, M. Langlais, and H. Malchow, "The Allee effect and infectious diseases: Extinction, multistability, and the (dis-)appearance of oscillations," American Naturalist, vol. 173, no. 1, pp. 72-88, 2009.

[25] N. Liu, J. Fang, W. Deng, and J. wei Sun, "Stability analysis of a fractional-order SIS model on complex networks with linear treatment function," Advances in Difference Equations, vol. 2019, no. 1, 2019.

[26] F. W. Zhang and L. F. Nie, "Dynamics of SIS epidemic model with varying total population and multivaccination control strategies," Studies in Applied Mathematics, vol. 139, no. 4, pp. 533-550, 2017.

[27] E. Okyere, J. Ackora-Prah, and F. T. Oduro, "A Caputo based SIRS and SIS fractional order models with standard incidence rate and varying population," Communications in Mathematical Biology and Neuroscience, 2020.

[28] H. El-Saka, "The fractional-order SIS epidemic model with variable population size," Journal of the Egyptian Mathematical Society, vol. 22, no. 1, pp. 50-54, 2014.

[29] M. Hassouna, A. Ouhadan, and E. H. El Kinani, "On the solution of fractional order SIS epidemic model," Chaos, Solitons and Fractals, vol. 117, pp. 168-174, 2018.

[30] A. Suryanto, I. Darti, and S. Anam, "Stability analysis of a fractional order modified Leslie-Gower model with additive Allee effect," International Journal of Mathematics and Mathematical Sciences, vol. 2017, no. 0, pp. 1-9, 2017.

[31] H. S. Panigoro and D. Savitri, "Bifurkasi Hopf pada Model Lotka-Volterra orde-fraksional dengan efek Allee aditif pada predator," Jambura Journal of Biomathematics, vol. 1, no. 1, pp. 16-24, 2020.

[32] H. S. Panigoro, A. Suryanto, W. M. Kusumawinahyu, and I. Darti, "A Rosenzweig-MacArthur model with continuous threshold harvesting in predator involving fractional derivatives with power Law and Mittag-Leffler kernel," Axioms, vol. 9, no. 4, p. 122, 2020.

[33] M. Moustafa, M. H. Mohd, A. I. Ismail, and F. A. Abdullah, "Dynamical analysis of a fractional-order ecoepidemiological model with disease in prey population," Advances in Difference Equations, vol. 2020, no. 1, p. 48, 2020.

[34] S. Othman and S. Othman, "Global stability of a fractional order sir epidemic model with double epidemic hypothesis and nonlinear incidence rate," Communications in Mathematical Biology and Neuroscience, pp. 1-15, 2020.

[35] R. Mondal, D. Kesh, and D. Mukherjee, "Dynamical analysis of a fractional order Rosenzweig-MacArthur model incorporating herbivore induced plant volatile," Chinese Journal of Physics, vol. 68, pp. 258-269, 2020.

[36] I. Petras, Fractional-order nonlinear systems: modeling, analysis and simulation. Beijing: Springer London, 2011, vol. 1.

[37] Y. Li, Y. Q. Chen, and I. Podlubny, "Stability of fractional-order nonlinear dynamic systems: Lyapunov direct method and generalized Mittag-Leffler stability," Computers \& Mathematics with Applications, vol. 59, no. 5, pp. 1810-1821, 2010.

[38] H.-L. Li, L. Zhang, C. Hu, Y.-1. L. Jiang, and Z. Teng, "Dynamical analysis of a fractional-order predator-prey model incorporating a prey refuge," Journal of Applied Mathematics and Computing, vol. 54, no. 1-2, pp. 435-449, 2017.

[39] C. Vargas-De-León, "Volterra-type Lyapunov functions for fractional-order epidemic systems," Communications in Nonlinear Science and Numerical Simulation, vol. 24, no. 1-3, pp. 75-85, 2015.

[40] J. Huo, H. Zhao, and L. Zhu, "The effect of vaccines on backward bifurcation in a fractional order HIV model," Nonlinear Analysis: Real World Applications, vol. 26, pp. 289-305, 2015.

[41] E. Ahmed, A. M. A. El-Sayed, and H. A. A. El-Saka, “On some Routh-Hurwitz conditions for fractional order differential equations and their applications in Lorenz, Rössler, Chua and Chen systems," Physics Letters A, vol. 358, no. 1, pp. 1-4, 2006.

[42] K. Diethelm, N. J. Ford, and A. D. Freed, "A predictor-corrector approach for the numerical solution of fractional differential equations," Nonlinear Dynamics, vol. 29, no. 1-4, pp. 3-22, 2002.

2020 by the Authors. This article is an open access article distributed under the terms and conditions of the Creative Commons Attribution-NonComercial 4.0 International License. Editorial of JJBM: Department of Mathematics, State University of Gorontalo, Jln. Prof. Dr. Ing. B. J. Habibie, Bone Bolango 96119, Indonesia. 


\section{Submit your manuscript at http://ejurnal.ung.ac.id/}

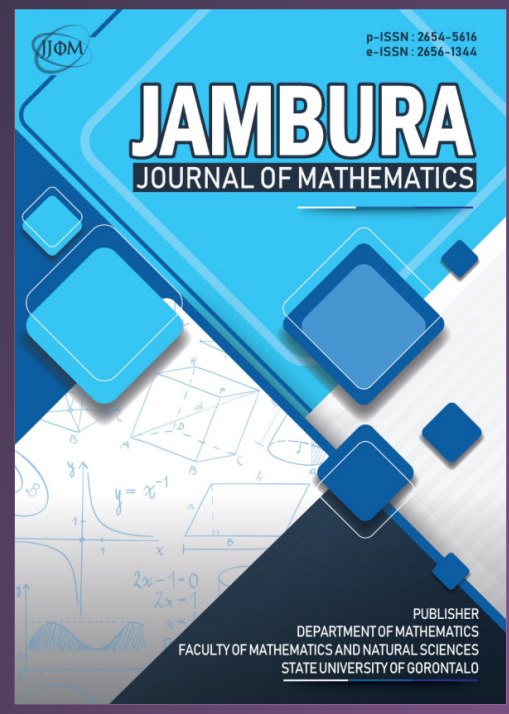

Jambura Journal of Mathematics

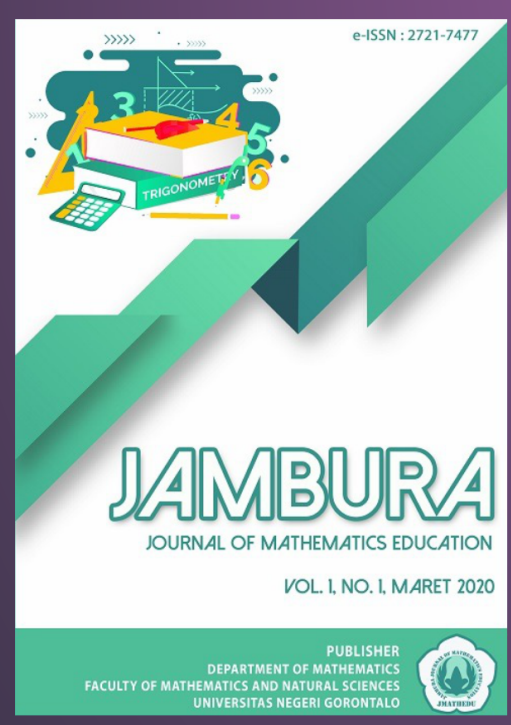

Jambura Journal of Mathematics Education
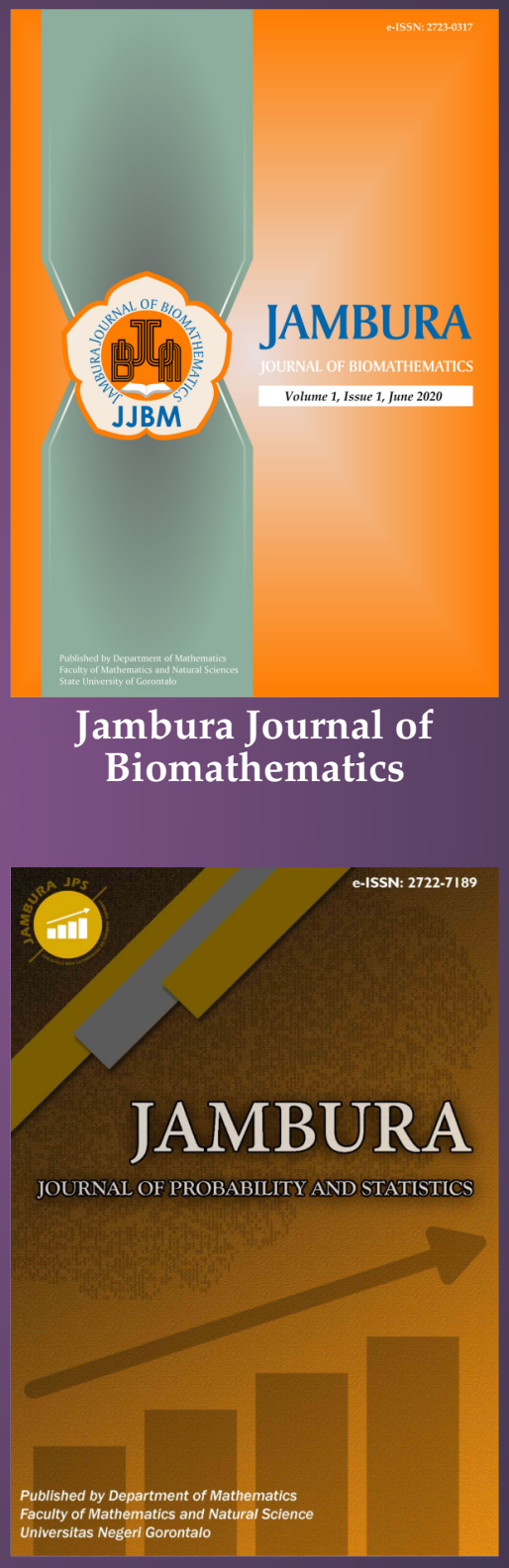

Jambura Journal of Probability and Statistics

Published by

Department of Mathematics

Faculty of Mathematics and Natural Sciences

State University of Gorontalo 